\title{
LOCALIZACIONES PARA UNA ESPACIALIDAD: TERRITORIOS DE LA MIGRACIÓN PERUANA EN SANTIAGO DE CHILE
}

\author{
LOCATIONS FOR A SPATIALITY: TERRITORIES OF PERUVIAN \\ MIGRATION IN SANTIAGO, CHILE
}

\author{
Alejandro Garcés $H .^{1}$
}

\begin{abstract}
El siguiente artículo aborda algunas transformaciones del espacio urbano de Santiago de Chile que son inducidas o producidas a partir del proceso de incremento del flujo migratorio peruano de los últimos 15 años. Desde una perspectiva etnográfica y a través de las biografías y trayectorias individuales de los migrantes y de la observación de sus usos y apropiaciones de los espacios urbanos, se ha detectado la configuración de éstos como locus proveedores de recursos para las nuevas comunidades migratorias. Estos lugares permiten tanto el acceso a informaciones claves para su reproducción económica, como la construcción de unos relatos del espacio y una memoria del mismo, que funcionan a su vez como fuentes de sentido para una nueva territorialidad. Las formaciones económicas de esta migración, y las diversas prácticas de reunión, asociación o aglomeración en los espacios públicos visibilizan la generación de un espacio urbano que es efecto de la actuación de distintos principios de localización espacial -algunos acotados físicamente, circunscritos a las fronteras de un barrio, unas calles o un local comercial, y otros virtuales, constituidos o descritos por los intensos movimientos y desplazamientos que caracterizan los flujos migratorios transnacionales-.
\end{abstract}

Palabras claves: migración peruana, espacio urbano, espacio público, transnacionalismo, comercio inmigrante.

This paper discusses the changes in urban space in Santiago, Chile caused by the substantial increase in Peruvian migration in the last 15 years. Using an ethnographic approach - with migrants' life stories, and observations of uses or appropriations of some urban areas-it was possible to see how Peruvian migrants have given shape to multifunctional loci which constitute resources for new migrant communities. These loci permit access to key information for economic reproduction, construction of spatial memory, and a source of meaning for a new territoriality. The economic formations of Peruvian migration and various practices of assembly, association or agglomeration in public places, makes visible a different construction of urban space. These novel urban forms result from the application of different principles of spatial landscapes. Some of these principles are physically confined, restricted to the borders of a neighborhood or a street or a shop, but others are virtual and constructed or described by the intense movements and displacements that characterize transnational migration flows.

Key words: Peruvian migration, urban space, public space, transnationalism, ethnic business, special landscapes.

Sin contar con una marcada tradición como espacio urbano receptor de flujos migratorios internacionales, la ciudad de Santiago de Chile ha sido escenario, en los últimos quince años, de una importante intensificación de su papel como destino de un movimiento migratorio de origen peruano. A partir de un trabajo de campo etnográfico desarrollado en dos espacios de la ciudad de Santiago en que se manifiesta la presencia concentrada de la migración peruana tanto a nivel residencial como en el ámbito comercial ${ }^{1}$, el artículo presenta tres formas de localizar la diferencia que la migración peruana introduce en la ciudad, localizaciones que en su interior anidan tanto dimensiones relativas a la reproducción social y económica del grupo migrante, como otras de orden simbólico, relacionadas con la construcción de sentidos de lugar y la producción de espacios para la denotación y estigmatización de la migración peruana en Santiago.

Los elementos que explican el desplazamiento de población peruana hacia Chile pueden responder a una diversidad de factores, pero existe consenso en señalar el mejoramiento de la situación económica y personal de los migrantes como la causa más importante en este sentido (Araujo et al. 2000; Stefoni 2002). De acuerdo a los datos del censo poblacional realizado en Chile el año 1992, el número de extranjeros nacidos en Perú alcanzaba las 7.649 personas, mientras que en 2002 esta cifra se elevaba a 37.860 personas (Martínez Pizarro 2003). Luego,

1 Instituto de Investigaciones Arqueológicas y Museo (IIAM), Universidad Católica del Norte. C/ Le Paige 380, San Pedro de Atacama, Chile. agarces@ucn.cl, ajgarces@gmail.com 
la concentración residencial de los peruanos por regiones en Chile resulta de singular importancia al señalarnos por una parte, que el 77,9 \% de ellos residen en la Región Metropolitana (Cano et al. 2009; Cano y Soffia 2009), y por otra, que Santiago (con 5.850 extranjeros peruanos) constituye el territorio municipal donde su presencia es más expresiva, seguido por el de Independencia (con 1.288 extranjeros peruanos) (Martínez Pizarro 2003). Este componente inmigrante en la ciudad de Santiago tiene una notoria visibilidad en el espacio urbano, y ya sea por los efectos de su inserción laboral o por sus particulares prácticas de ocio y socialización comunitaria, la migración peruana transforma y se apropia de la ciudad. Precisamente en las comunas arriba reseñadas la migración peruana asume de manera privilegiada esta forma concentrada que da lugar a una particular y visible presencia en el espacio público y a una pujante emergencia de comercios en principio orientados hacia sus connacionales. Pues bien, ¿cómo puede caracterizarse la actividad que los migrantes desarrollan en estos espacios? ¿qué funciones cumple en el marco de las experiencias migrantes en destino del flujo migratorio? ¿de qué manera en estos espacios adquieren vigencia elementos propios de los lugares de origen? ¿de qué modo estas concentraciones son usadas para denotar y estigmatizar al colectivo migrante?

\section{Primera Localización: Espacio-Recurso}

La comprensión de los lugares de concentración residencial y comercial de la migración como espacio-recurso significa entender que tanto la actividad económica así como los usos del espacio público que allí se manifiestan resultan claves en la reproducción social y económica del colectivo migrante, al tiempo que configuran el escenario urbano para la articulación de sus redes migratorias. En este sentido, nos inspira la noción de espacio/ recurso entendido como "medio de supervivencia, estímulo a su utilización, ocasión de crecimiento, pero también de riesgo... en el concepto de recurso está implícita la utilización de un potencial del que se puede disponer..." (Signorelli 1999:53-54). La actividad comercial resulta sin duda uno de los mecanismos localizadores más relevantes del espacio urbano construido por la migración peruana en el centro de Santiago, y constituye al mismo tiempo un factor de atracción para el conjunto del colectivo residente en la ciudad. A diferencia de otras urbes con presencia migratoria de diversas procedencias nacionales - donde la actividad comercial migrante tiende a extenderse por el conjunto del espacio urbano (Garcés 2005; Serra 2008)- se puede plantear que en nuestro caso los comercios inmigrantes peruanos se presentan de manera muy concentrada en las zonas centrales de la ciudad. En el contexto de las migraciones internacionales se ha aceptado comúnmente la definición de enclave como la concentración en un espacio físico (generalmente en un área metropolitana) de firmas o empresas étnicas que emplean una proporción significativa de trabajadores de una misma minoría (Wilson y Portes 1980). Tratándose de una agrupación de población de un determinado grupo étnico fuera de su territorio de origen, ha sido objeto de discusión si esta concentración puede ser definida en términos económicos o residenciales, esto es, si exclusivamente se desarrolla la actividad económico-comercial en la zona definida como enclave, o si ésta se definiría a partir del emplazamiento residencial del grupo migrante (Portes y Jensen 1989). Sin embargo, para el caso de la migración peruana en Santiago, la concentración abarca ambas dimensiones, encontrándonos tanto con migrantes que residen y desarrollan su actividad económica en estos lugares, como otros que residiendo en diversas zonas de la ciudad, localizan su actividad comercial en estos espacios específicos.

Si observamos los rubros en que se inscriben los comercios que estudiamos, vemos que se trata de una oferta de servicios y bienes que pueden relacionarse con lo que se ha venido a denominar necesidades "culturalmente específicas" o "intrínsecas" de las poblaciones migrantes (alimentación, religión, ocio) o aquellas que se derivan de su condición de foráneos (Buckley 1998). Sin embargo, antes de entender de manera mecanicista la emergencia de estos emprendimientos como respuesta a una supuesta necesidad cultural, parece más plausible comprender estos emprendimientos como formaciones condicionadas por la actuación de las redes migratorias, entendidas estas últimas como "conjuntos de vínculos interpersonales que conectan a migrantes, antiguos migrantes y no migrantes en su área de origen o de destino a través de los lazos de parentesco, amistad y comunidad de origen compartida" (Massey et al. 1998:229). Son este tipo de vínculos los que presentan una articulación específica en el caso de los emprendimientos comerciales desarrollados por los migrantes. En este sentido 
Werbner propone examinar las múltiples fuerzas que generan pequeños negocios interdependientes dentro de un enclave étnico. Por un lado, las dinámicas internas, relacionadas con el crecimiento y extensión familiar, y por otro, la existencia de unas fuerzas externas tanto económicas como demográficas, relacionadas con el incremento de la competencia y el influjo de los inmigrantes recién llegados al nicho o enclave (Werbner 1987), en el entendido de que ambas fuerzas cooperan en la consolidación de estos emprendimientos. Además de la existencia de un nicho de negocio en la sociedad de destino, tras la formación de muchas de estas empresas y del inicio mismo de los proyectos migratorios, se encuentran las relaciones de parentesco, de amistad, y el hecho de compartir el lugar de origen como elementos fundamentales.

En el caso de Santiago identificamos tres rubros de negocio que destacan notoriamente entre el colectivo migrante peruano: los centros de llamados telefónicos e Internet, los restaurantes y cocinerías de comida peruana, y los puestos de venta de productos de alimentación peruanos. Los primeros son locales comerciales que intentan prestar la más amplia gama de servicios relativos a la comunicación con las localidades de origen en Perú, incluyendo en algunos casos el servicio de envío de dinero. Se trata del rubro de negocio más extendido en la ciudad y su localización acompaña la concentración residencial peruana en algunas zonas del municipio de Santiago e Independencia, fundamentalmente. Los restaurantes peruanos constituyen una actividad de importante crecimiento durante la última década. Pueden presentar una gran diversidad interna, desde aquellos de más alto standing ubicados en los municipios más ricos de la ciudad, algunos de nivel medio en el municipio de Santiago, y aquellos que hemos denominado "cocinerías", por lo general más modestos en términos de espacio de funcionamiento $y$ presentación gastronómica.

Si bien nuestra investigación ha podido detectar la introducción de empresarios chilenos en estos negocios -lo que a su vez disloca en gran medida la categoría de enclave que suele asentarse sobre una premisa de monoetnicidad en el espacio (Garcés 2007, 2010)-, lo más extendido entre la gran mayoría de emprendimientos regentados por migrantes es la gestión familiar de los negocios. Los recursos internos de la comunidad expresados en la solidaridad y el trabajo familiar adquieren un importante valor económico en el que en parte puede basarse la competitividad de estos negocios, sobre todo considerando la extensión de las jornadas laborales requeridas por su público. Quienes regentan estos comercios se valen de familiares y amigos para el desempeño de las distintas tareas administrativas, de provisión de bienes y de atención de público. Sin embargo, no es posible explicar el surgimiento de esta particular formación económica exclusivamente en términos de la dinámica interna del grupo migratorio, considerando sólo el tipo de necesidades que se satisfacen y la forma en que se dispone de los recursos familiares para gestionar los distintos aspectos de la actividad comercial (Light y Gold 2000). Entran a tallar también condicionantes de orden individual y relativos al contexto en que tienen lugar estos emprendimientos (Waldinger et al. 1990).

Las historias migratorias recogidas en entrevistas con comerciantes peruanos nos hablan de diversas trayectorias en los procesos de formación de estas pequeñas empresas, siendo predominante un movimiento que va desde el empleo en sectores de baja cualificación hacia estas nuevas formas de emprendimiento comercial ${ }^{2}$. Los comerciantes peruanos que entrevistamos se empleaban antes en el servicio doméstico (las mujeres), y en la construcción y otros empleos asalariados (los hombres), tendiendo a ser menos relevantes las experiencias anteriores como autónomos o empleados en el sector comercial. Estas trayectorias previas resultan singularmente importantes al construir los capitales sociales con que cuentan los migrantes al momento de desarrollar sus emprendimientos (Light y Gold 2000). Es particularmente notorio el que frente a las anteriores experiencias de trabajo asalariado, el trabajo autónomo e independiente emerge con una alta valoración en el discurso de los migrantes,

Yo llegué a Santiago digamos aproximadamente hace ocho meses, por intermedio de un amigo que tiene un negocio acá de productos peruanos. Él me dio la idea, él me dijo al menos acá hay un futuro... Así que me dijo 'sabís qué? Mejor vente para acá...' como ya él conoce. Bueno le digo, me iré para allá...porque me vi seguro con esto, por lo que él me dijo, por lo que él ha logrado acá, o sea yo quiero lograr eso. Me entiendes? Yo vengo acá con esa meta, con esa mentalidad... Ya yo estaba cansado de recibir órdenes, de madrugar al 
trabajo, porque es matadito el trabajo. Yo dije tengo un capital y yo quiero tener lo mío, ser independiente (HO, comerciante peruano de la Vega Central).

A partir de este relato puede ser discutida la primacía de las explicaciones culturalistas que han dominado en algunos estudios sobre economía étnica. Según estos enfoques, los atributos culturales de ciertos grupos minoritarios explican y determinan la tendencia a la inversión y a la creación de negocios propios, anulando las condiciones materiales o estructurales que constituyen el contexto donde se desarrollan estos emprendimientos comerciales (Cebrián de Miguel y Bodega 2002). Los discursos de los comerciantes revelan precisamente que más allá de la aproximación culturalista que fija una predisposición en el grupo, lo que hay es una alta valoración del trabajo independiente y la posibilidad de acceder a mejores ingresos. Frente a la visión culturalista de estos espacios como lugares de explotación de las nuevas minorías (Bonacich 1973), se observa que estos emprendimientos posibilitan la movilidad social de los migrantes y sus familias, mejorando las condiciones de vida de sus descendientes (acceso a estudios universitarios y mejora de la cualificación de las segundas generaciones) y los procesos de creación de riqueza y de empleo en el ámbito urbano (Wilson y Portes 1980; Cebrián de Miguel y Bodega 2002; Beltrán et al. 2007). Frente a las escasas oportunidades de movilidad social que ofrece el mercado del trabajo local, las economías étnicas de lo peruano en Santiago se constituyen tanto en un espacio de aprendizaje como en una vía de ascenso e inserción urbana.

Considerando estos aspectos, las perspectivas "interaccionistas" sobre el comercio inmigrante nos proponen una lectura integrada de las comunidades de migrantes y de la estructura local de oportunidades en que se insertan (Waldinger et al. 1990). Se discute en este sentido la presentación de estos emprendimientos en una suerte de vacío institucional, sin visibilizar las condicionantes económicas y situacionales en que tienen lugar estas economías o su "reacción al bloqueo de oportunidades para los migrantes en el mercado de trabajo; de manera que la autoocupación se erige como una estrategia de supervivencia vinculada a los lazos de solidaridad existentes dentro de la comunidad" (Solé y Parella 2005). La precariedad del empleo en la construcción y en el servicio doméstico, la situación de irregularidad en la que muchos migrantes desarrollan su actividad laboral (lo que impone dificultades en la formalización de contratos de trabajo, cuando no unas condiciones laborales abusivas), son todos elementos que conforman el contexto de la inserción social de los migrantes peruanos en la ciudad. Estas cuestiones combinadas con las condiciones internas del grupo-expresadas en el crucial papel de las redes sociales y el trabajo familiar, en la existencia de un conjunto de necesidades específicas y un mercado cautivo (Beltrán et al. 2007; Kloosterman et al. 1999)- dibujan la interconexión entre economía y etnicidad que en gran medida explica la emergencia del comercio inmigrante.

La apropiación que implica estos nuevos negocios de la migración no puede reducirse a la mera actividad comercial que les caracteriza, sino que además presenta un cierto correlato en los usos del espacio público de la calle, y que en primera instancia también cooperan en su configuración como espacio-recurso. La presencia de los migrantes puede ser descrita como permanente puesto que puede observarse durante todos los días de la semana, manteniendo un relativo equilibro en cuanto a la presencia masculina y femenina, con la excepción del fin de semana en que se intensifica de modo notable la presencia de las mujeres. La intensiva ocupación de estos espacios los fines de semana por parte de mujeres peruanas se correlaciona con los días de descanso con que cuentan, cuando se dirigen al centro para reunirse con amigos o familiares, comer platos peruanos en las cocinerías y restaurantes, enviar dinero y comunicarse vía Internet o teléfono con sus lugares de origen.

En este sentido, es destacable el que las apropiaciones de estos espacios por parte del colectivo peruano se encuentran necesariamente ligadas a las condiciones de trabajo o a los nichos de empleo a los que estas poblaciones acceden. El desplazamiento por la ciudad estimulado por la ubicación del lugar de empleo por una parte, y la localización de los lugares de encuentro y acceso a diversos servicios propios de la migración por otro, desdibujan la correlación isomórfica entre la forma espacial y las relaciones sociales en la ciudad (influencia que proviene de la Escuela de Chicago). En perspectiva crítica a esta correlación Martínez Veiga plantea la actuación, en el caso de los migrantes trabajadores del servicio doméstico, de una segregación espacial que no operaría por la distancia física que se establece entre los grupos, sino que por el establecimiento 
de unas relaciones sociales que, articuladas en el trabajo, separan o aíslan a unos individuos de otros, invisibilizándolos respecto del colectivo. Este aislamiento entre unas trabajadoras y otras es resuelto de manera colectiva por el grupo migrante a través de los llamados 'fenómenos de aglomeración compensatoria', entendidos como centros de reunión, al aire libre o no, donde los miembros de un determinado colectivo se congregan (Martínez Veiga 1999) (Figuras 1 y 2).

Así, las distintas prácticas de utilización del lugar por parte del colectivo peruano en Santiago -y su capacidad para articular los flujos de información y acceso a servicios de la red social migrante (Garcés 2006; Peñaranda et al. 2011)- dotan al espacio público de una gran centralidad en la reproducción económica y social de estas poblaciones. Sin embargo, es la misma idea de aglomeración, o la forma "compensatoria" en que se actualiza, lo que nos da pistas para establecer la porosidad de las fronteras de estos espacios, su desbordamiento y la articulación de un nuevo principio de localidad soportado ahora por la movilidad en el conjunto de la ciudad. La tensión entre la fuerza centrípeta de las relaciones sociales del grupo representadas en la aglomeración y la agregación por una parte, y la fuerza centrífuga expresada en el aislamiento (del trabajo doméstico en este caso) por otra, hace emerger a los migrantes en sus desplazamientos por la ciudad. Así, estos espacios de concentración de los migrantes peruanos no devienen lugares cerrados sobre sí mismos, acotados por el conjunto de relaciones y funciones actualizadas al interior de sus fronteras. Los espacios de la migración peruana provocan y son parte de una territorialidad que adquiere vida en el conjunto urbano.

\section{Segunda Localización: Sentidos de lugar y Espacio Transnacional}

Una segunda localización de la experiencia migrante peruana en Santiago es proveída a partir de la configuración de sus espacios, con el conjunto de dinámicas comerciales y prácticas de ocupación del espacio público que les caracterizan, como locus identitario para los grupos migrantes. No nos estamos refiriendo a la construcción de una identidad esencializada, configurada en un acervo acotado de rasgos, sino más bien a unos "sentidos de lugar" que trabajan a partir de materiales diversos, con elementos de origen y destino del flujo migratorio, y que en definitiva están compuestos por un conjunto de interacciones sociales que enlazan a las personas a unos determinados territorios, como un proceso más que un conjunto de cosas (Massey 1994:155).

Entendemos la generación de una territorialidad migrante en la ciudad como una construcción que es consustancial a la conformación y visibilidad del grupo migrante, y que consolida un principio de identidad colectiva. Esta territorialidad requiere la consolidación en el tiempo de una serie de prácticas que le caracterizan, y que en esa medida le historizan. Así pues, Tarrius aborda lo migratorio como una experiencia de movilidad que articula de manera específica las categorías de espacio, tiempo e identidad, y donde las territorialidades migrantes operarían a través de un "espacio-tiempo de los consumos repetitivos, a menudo cotidianos, de los lugares y de la reactivación de los vínculos de identidad", una dimensión constituida por las secuencias temporales como caminos usados para realizar actividades, señalando proximidades sociales y espaciales fundadoras de la cohesión del grupo y de las vecindades. En definitiva, unos itinerarios que identifican secuencias consecutivas de la vida social, unos "ritmos sociales" (Tarrius 2000). Los discursos de los migrantes peruanos apuntan a este proceso de historización de los espacios; hacia la construcción de una memoria colectiva respecto de los mismos que entienden este tipo de ocupaciones como la repetición de una práctica cultural que viene de antaño asociada a los movimientos migratorios en origen (Golte 1999).

De este modo, la producción de estos discursos y prácticas de identidad se valen de mecanismos o materiales que vinculan los territorios de origen y destino de la migración, desbordando nuevamente la producción de una localidad restringida a unos espacios fronterizados: los espacios de concentración. Nos encontramos con unas prácticas y unos discursos que espacializan la experiencia migrante, pero que lo hacen valiéndose de la formación de un campo transnacional. Siguiendo la perspectiva de Glick Schiller et al. (1992), podemos entender la formación de este campo como una "red de relaciones sociales", donde las formaciones comerciales y las economías étnicas de la migración serían paradigmáticas, constituyendo el motor de una continua circulación y nuevo establecimiento de personas en destino. Asimismo, el campo transnacional se entiende también como la "circulación de cosas o bienes" como elemento central en la construcción 

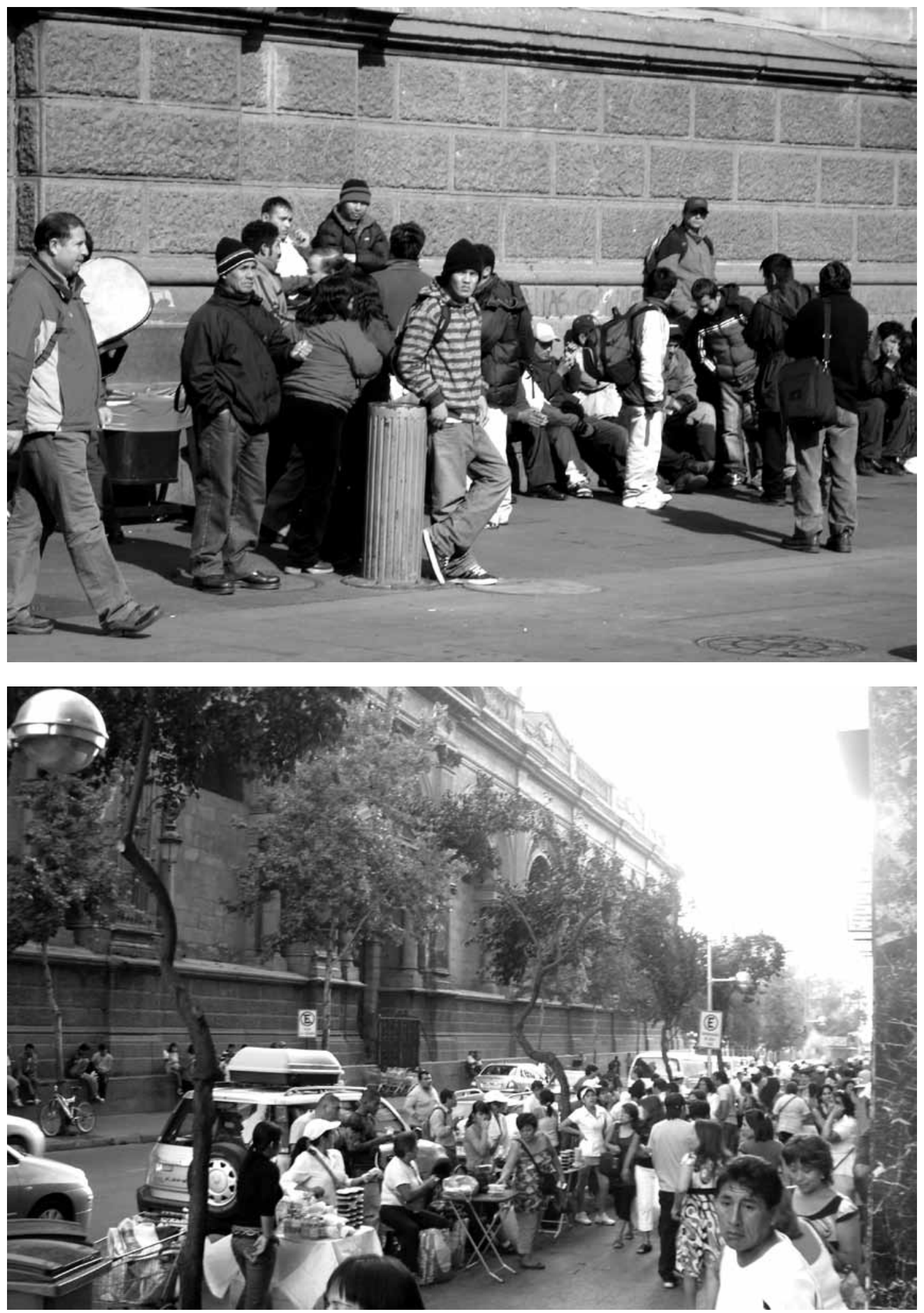

Figuras 1 y 2. Aglomeración de migrantes peruanos en Santiago.

Agglomeration of Peruvian migrants in Santiago. 
del paisaje de "lo migrante" en la ciudad (Glick Schiller et al. 1992). Aquí los objetos pueden servir como recuerdos, generando continuidad a través de espacios y tiempos diversos, pueden llegar a asociarse con personas y lugares geográficamente distantes, y pueden cooperar en la elaboración de unos "relatos de espacio" " a través de los cuales las personas adquieren y expresan conocimientos compartidos de lugares lejanos y de sus habitantes, permitiendo que una población dispersa constituya comunidad (Boruchoff 1999). Si el lugar coloca a las cosas en relaciones de coexistencia, los objetos permiten la producción de unos relatos de espacio que se desbordan, que salen de sus límites físicos para producir una nueva espacio-temporalidad, un desborde para una nueva localización. Lo narrado por una migrante peruana acerca de sus recorridos por uno de estos espacios es sintomático de este tipo de construcciones que van desde las cosas a la yuxtaposición de los lugares:

... por los rostros, por los olores, por las cosas que veo, es como eso... sí, en las noches alguna vez he pasado por acá y se sienten todos los olores. En la mañanas, las comidas. Es fuerte o sea, no sé si para las otras personas tendrá el mismo significado pero a mí me llama mucho este espacio. Cuando yo llegué mi mamá me dijo 'te voy a llevar a conocer un lugar que te va a hacer sentir que no estás en otro país'... Y me trajo, vinimos en la noche por acá. Y dije 'es cierto!'... (ME, miembro de asociación cultural).

Para el caso específico que nos ocupa, la presencia de estos objetos operan en la recreación de un sentido de lo nacional, instrumental si se quiere, pero efectivo como proyecto. Los bienes o cosas como hitos identitarios constituyen marcas que permiten evocar fácilmente territorios diversos, comunicando o enlazando contextos de origen y recepción ${ }^{4}$ (Figuras 3 y 4 ).

Nos encontramos entonces con unos espacios urbanos de la migración peruana que son la base o el soporte material de una dinámica transnacional, espacios cuya práctica se valen del recurso al movimiento, al desplazamiento de personas, cosas e imaginarios (Appadurai 2001). Una de las comprensiones acerca del transnacionalismo más extendidas y sobre todo más operativas es aquella que lo entiende como el conjunto de ocupaciones y actividades que requieren contactos sociales regulares y sostenidos a través del tiempo y de las fronteras nacionales para su implementación (Portes et al. 1999). Si bien la novedad de la intensificación del fenómeno migratorio peruano en Santiago puede obstaculizar aún la consolidación del carácter regular y sostenido de los vínculos entre origen y destino que supone la perspectiva transnacional, el fenómeno en Santiago sí permite comenzar a observar algunos trazos en este sentido ${ }^{5}$. El desenvolvimiento de los comercios inmigrantes -su actividad incesante, las prácticas a que dan lugar-, opera, por una parte, hacia la fijación de un espacio o hacia la delimitación de sus fronteras, y por otra, hacia la articulación de diversos movimientos o flujos, ya sea de personas como de cosas.

\section{Tercera Localización: de Espacio Transnacional a Clausura Nacional}

Nuestra observación de estos espacios de concentración de la sociabilidad peruana en Santiago hacen emerger una tercera forma de notar su espacialidad, de localizarles. En cierta forma, esta última opera en dirección contraria a la conformación de fronteras "porosas" que supone la dinámica transnacional, dado que nos referimos a las actuaciones que desde la sociedad receptora del flujo migrante refuerzan el confinamiento y el anclaje espacial de la comunidad migrante. Nuestra tercera localización de los espacios peruanos dibuja por lo tanto una clausura nacional.

Los usos de la ciudad actualizados por los migrantes peruanos escenifican una cierta y concreta sociabilidad migrante al interior de la urbe, obstaculizan la actualización de un espacio público entendido como un escenario organizado en torno al anonimato. Este "espacio anónimo" que sería condición de cualquier sociabilidad democrática (y que en principio intenta ser cautelado en la planificación urbana) pone paréntesis lo específico a las identidades de cada individuo, produciendo lo urbano como una práctica del movimiento, de la circulación, de las trayectorias individuales (Delgado 1999). Uno de los espacios de la migración peruana con que trabajamos se ubica en la zona céntrica de la ciudad, lugar de intenso movimiento y que podría ser caracterizado como espacio para la circulación, escenario ideal para la operación del principio definitorio de lo urbano que señalaba Goffman, la 


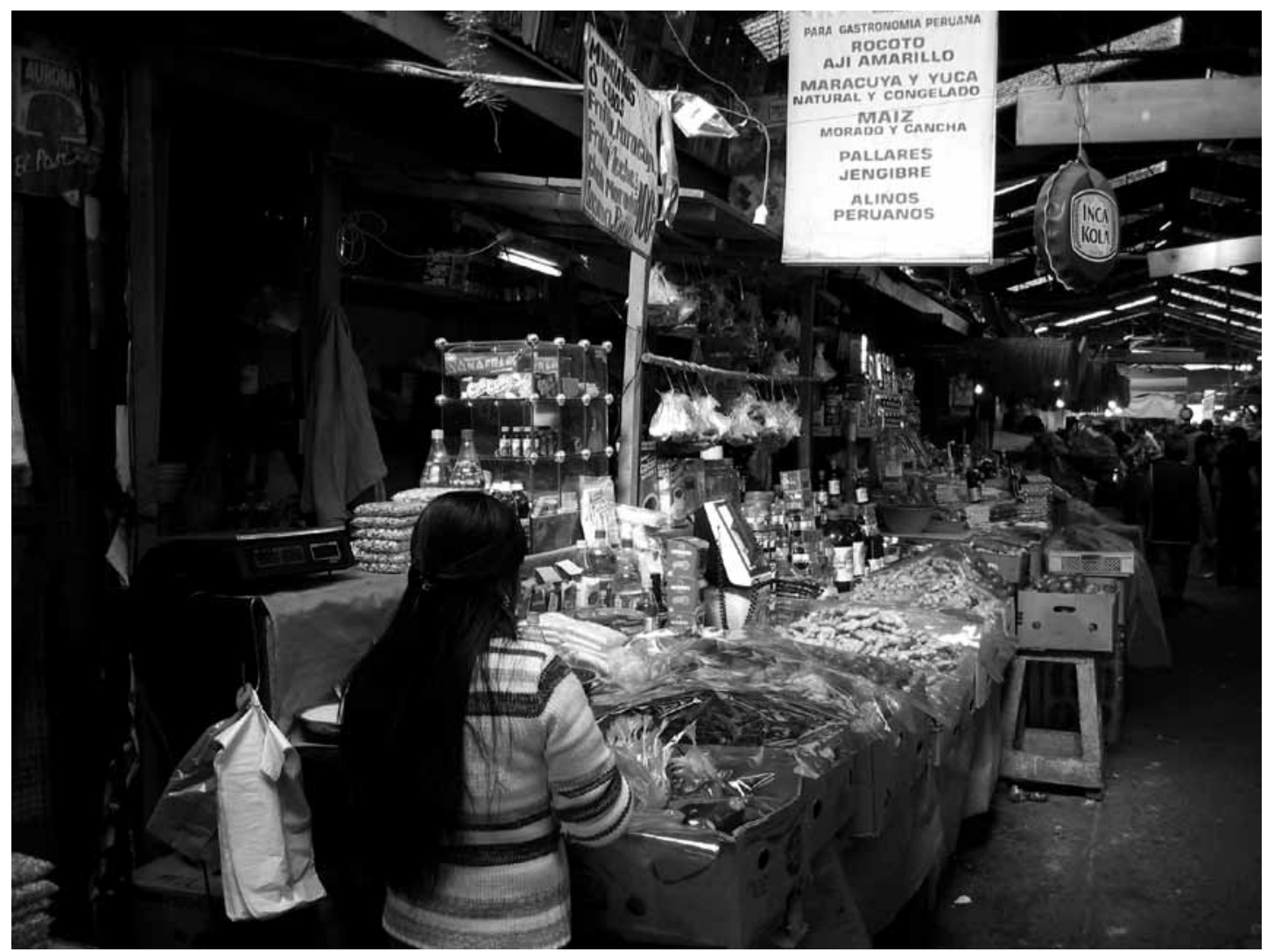

Figura 3. Comercio de productos peruanos importados en la Vega Central.

Trade of Peruvian goods in "La Vega Central" (central market).

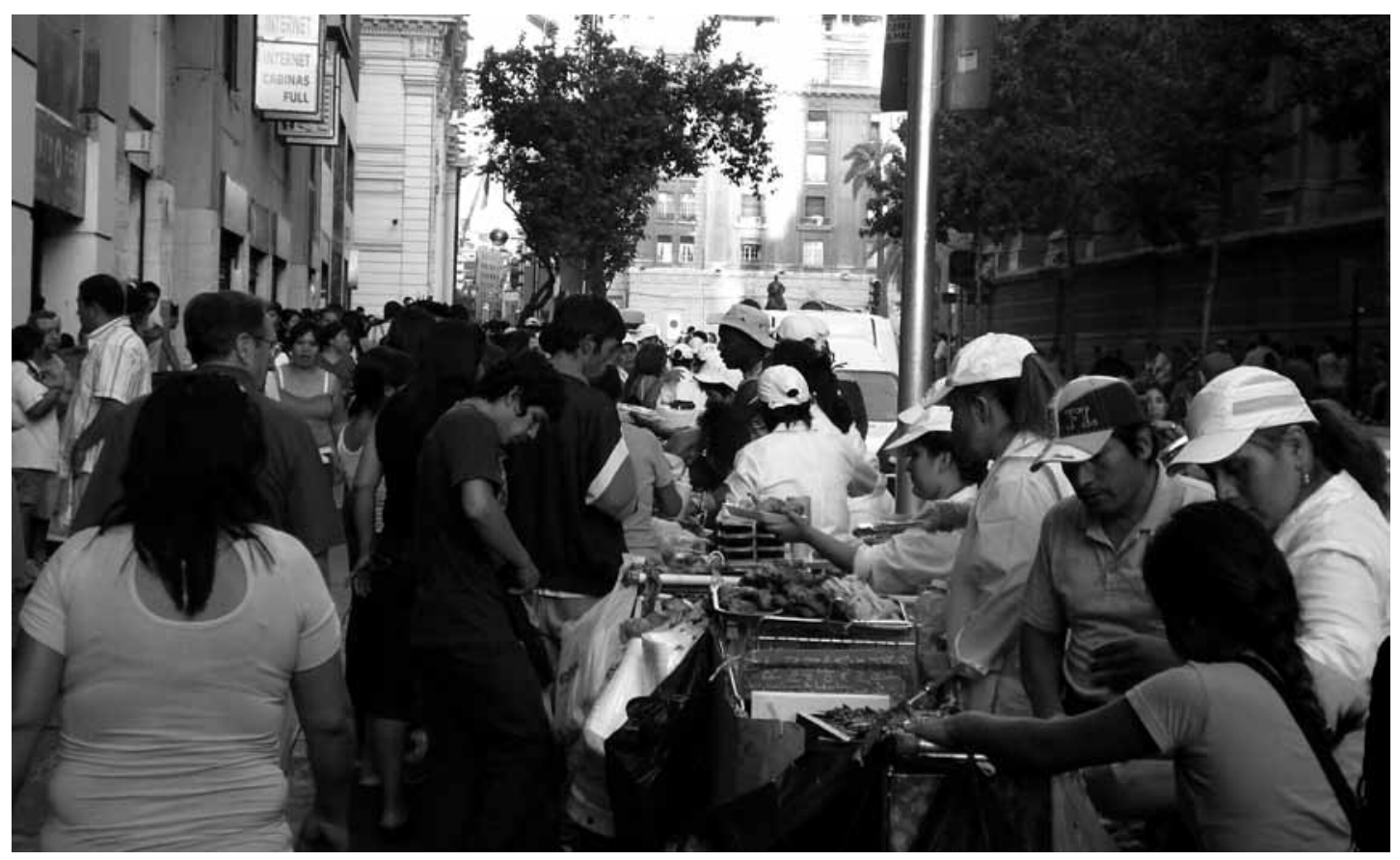

Figura 4. Venta callejera de comida peruana preparada en el centro de la capital.

Downtown street vending of Peruvian food. 
“desatención cortés" (Delgado 2007). El entorno construido por los migrantes peruanos parece operar precisamente en sentido contrario. El carácter recurrente de los usos del espacio descritos, al mismo tiempo que el conjunto de relaciones cara a cara, familiares y/o comunitarias que se articulan en su interior, apuntan a la derogación de ese anonimato, a la configuración de una sociabilidad que tiende hacia una alta estructuración de sus relaciones, y donde la membrecía descansa fuertemente en la identidad (para empezar nacional) de los participantes. Nos encontramos ante unas prácticas de ocupación del espacio urbano, a unos conflictos que surgen a partir de la copresencia de lo migrante y lo autóctono, y ante la enunciación de unos discursos que anclan lo peruano a un territorio específico de la ciudad, elementos que inhiben la posibilidad de fijar una urbanidad a priori o anterior, aséptica, regida por el anonimato.

Tenemos entonces unos espacios que por efectos de la apropiación en curso configuran una diferencia y legitiman unas presencias y unas prácticas específicas. Producen así un particular "disciplinamiento", en la medida en que articulan un principio de clausura foucaultiano, esto es, la especificación de un lugar heterogéneo a los demás y cerrado sobre sí mismo. "Como arte de la distribución de los individuos en el espacio se realiza por la técnica de otorgar a cada individuo un lugar y a cada emplazamiento un individuo" (Foucault 2002). Sin embargo, este disciplinamiento por la vía de los usos y ocupaciones de la experiencia migrante entra en contradicción con el sentido de "espacio disciplinado" pretendido en la planificación urbana, aquel que legitima unos usos con anterioridad a la producción de lo urbano entendido como la calle o aquello que se ejecuta o despliega en ella (Delgado 2007).

Lo que se despliega en estos espacios es precisamente la anomalía de una "otra urbanidad", la anomalía de lo migrante. La fuerza disciplinaria de la planificación urbana trabaja desde un espacio representado o concebido, desde el cual pretende obtener una cierta legibilidad, una iluminación de zonas oscuras o la obtención del milagro de una inteligibilidad absoluta (Delgado 2007). Este espacio así disciplinado intenta "anular los efectos de las distribuciones indecisas, la desaparición incontrolada de los individuos, su circulación difusa, su coagulación inutilizable y peligrosa; táctica de antideserción, de antivagabundeo, de antiaglomeración" (Foucault 2002). Encontramos pues la conflictividad que introduce el carácter "indisciplinado" de las prácticas que organizan los espacios migrantes, donde por ejemplo destacan las dinámicas de "aglomeración compensatoria" que antes describimos. Una aglomeración que destruye las líneas de circulación por el espacio, o más bien las lógicas de esa circulación que se suponen consustanciales al espacio público. Los usos migrantes del espacio hacen opaca la disciplina que se constituye para conocer, dominar y utilizar; y al hacerla ilegible producen un espacio indisciplinado.

La discontinuidad que introduce la presencia migrante en el espacio urbano resulta particularmente visible en determinados lugares de la ciudad, donde se observa una potente concentración residencial y comercial. Una vez notada esta presencia, se articulan e imponen sobre ella un conjunto de dispositivos que intentan fijar o caracterizar su diferencia. Uno de ellos refiere a lo que podríamos denominar "criminalización del espacio", manifestado a través de un encuadre de actuaciones. Por un lado, tenemos aquellas de carácter institucional, referidas a la acción de distintos órganos del aparato público que contribuyen a describir el carácter "conflictivo" de las actividades y la presencia migrante, destacándose el "hostigamiento" de que son objeto los comerciantes extranjeros en lo relativo a la salubridad de restaurantes y cocinerías, y a la regularidad o formalidad de sus emprendimientos. Asimismo, la continua presencia policial en las calles y la realización de "redadas" para la detención de personas en situación de residencia irregular constituyen mecanismos que cooperan en la demarcación de un territorio específico en la ciudad, del mismo modo que construyen significados sobre el espacio y sus ocupantes.

Se suma a esta operación una actuación que no cuenta en primera instancia con una expresión material en el espacio urbano. Nos referimos a la producción estadística de informes de análisis relativos a la delincuencia en la ciudad, y que posteriormente fundamentan determinadas políticas de seguridad pública. Las estadísticas de denuncias de delitos de mayor connotación social (DMCS) ${ }^{6}$ permiten describir un aumento sostenido de estas denuncias y detenciones ${ }^{7}$, y localizar zonas específicas de la ciudad en las que su ocurrencia es más frecuente (áreas de concentración delictual), mostrando cómo los lugares en que se produce esta intensificación tienden a coincidir inexorablemente con los espacios donde se reproduce la sociabilidad migrante 
que describimos. Así, se observa que las denuncias y detenciones intensifican su frecuencia en las cercanías de las zonas en que se ubican comercios peruanos de telefonía, Internet, restaurantes y discotecas. La criminalización del espacio se expresa en la construcción de unas "cartografías" del delito protagonizadas por la presencia migrante.

Otro dispositivo que opera en la fijación de las diferencias que imponen las apropiaciones del espacio urbano por parte de la migración peruana, dice relación con la higiene y/o salubridad. Hemos señalado la gran visibilidad que el comercio ambulante tiene en el espacio de la calle, especialmente la venta de comidas preparadas que se convirtió en objeto de señalamiento por parte de las autoridades locales y de la población en general. La descripción hecha por un comerciante chileno del centro resulta ilustradora al respecto:

Lo que sí no sé qué piso usaría yo, porque son muy cochinos, muy cochinos. O sea, la inmundicia que dejan sábado y domingo es impresionante. Porque estas comidas que las venden en esas cosas de polietileno, las tiran ahí no más... no hay mucho basurero. Pero últimamente les habían puesto estos contenedores grandes... Yo creo que tendrían que darles patente en locales establecidos pero por ahí cachai? $\mathrm{O}$ darles la oportunidad de que vivan su realidad, si a ellos les gusta la comida en la calle po.... (M. comerciante chileno en calle Catedral, marzo 2009).

Resulta manifiesta la relación paradigmática establecida entre segregación espacial, minoría étnica e higiene, relación pesquisable en la literatura científica para momentos históricos y zonas geográficas diversas (Anderson 1987; Murunga 2005; Nightingale 2006). No queremos plantear con esto la necesidad de que los espacios migrantes localizados en el centro de la ciudad respondan solamente a un proceso de segregación espacial ${ }^{8}$, sino más bien nos interesa la articulación del mismo principio mediante el cual la higiene opera como marcador de las diferencias entre los grupos nativos y extranjeros, como ideología que permite mantener la separación entre unas poblaciones y otras, como un "proceso metonímico de conversión de los grupos étnicos en algo insalubre en sí mismos" (Martínez Veiga 2008). De lo que se trata en definitiva es de la construcción de un estereotipo (lo antihigiénico, lo insalubre) que se ancla al espacio, que se localiza y coopera en su reproducción como hecho social.

Los distintos mecanismos, actividades y discursos que localizan y caracterizan lo migrante -su indisciplinamiento, su criminalización, su insalubridad- no constituyen fuerzas que actúan únicamente como una denotación de lo local/autóctono respecto de lo migrante, en esa unívoca dirección. También se observa el uso de estos discursos por los propios migrantes a efectos de una diferenciación y jerarquización interna entre los grupos que componen la migración peruana en Santiago de Chile. Con todo, lo que estos discursos conllevan es la consolidación de unas fuerzas que determinan y fijan al grupo social migrante, que lo clausuran en una relación isomórfica entre identidad y espacio, o lo que es lo mismo, nacionalizan el espacio.

\section{Conclusión}

Los espacios de concentración de la migración peruana en Santiago de Chile inauguran o imponen nuevas formas de organización espacial que se superponen a las existentes en la ciudad. Dislocando o desbordando las sanciones territoriales que organizan las soberanías de los Estados-nación, las formas comerciales que desarrollan los migrantes, los usos y funcionalidades de sus espacios públicos, la circulación de personas y el flujo de mercancías que sostienen y estimulan, confluyen en la generación de una economía y una espacialidad que conecta territorios supuestamente escindidos unos de otros, esparcidos, aislados.

Planteamos específicamente la forma en que estos locus configuran un espacio-recurso para la reproducción del colectivo migrante, al mismo tiempo que sus comercios resultan centrales a los efectos de la construcción de una dinámica transnacional. Por una parte, la venta de productos importados desde los países de origen permite articular un flujo de bienes que se sostiene a partir de un contacto continuo con algunas localidades en origen. Por otra, la consolidación de los emprendimientos comerciales inmigrantes configura una suerte de motor para la circulación de las personas, en particular de familiares y amigos que inician sus proyectos migratorios. Asimismo, se ha observado cómo el espacio público de la calle y los centros de ocio (discotecas y restaurantes) de la migración peruana en Santiago de Chile van seleccionando y 
poniendo en circulación elementos propios del lugar de origen (ornamentaciones, músicas, comidas) resignificándolos, construyendo de este modo un "campo legítimo de lo peruano" en el nuevo espacio urbano y sancionando un imaginario que "certifica" una comunidad migrante. Luego, la territorialidad que emerge a partir de la experiencia migrante, expresada como hemos visto en el espacio de la ciudad, entra en conflicto con las estructuraciones, disposiciones, regulaciones propias del aparato del Estado y de la planificación urbana.

Así, con los materiales diversos a los que es posible acceder en un mundo globalizado, los grupos migrantes forman una nueva "cultura urbana", esto es, un proceso de creación y reelaboración a partir del contacto con los distintos desarrollos y conocimientos de otros grupos y sociedades del globo (Golte 1999). Se trata de una reelaboración de las distintas prácticas culturales en el nuevo contexto de la ciudad de recepción, del mismo modo que se sostienen nuevas lógicas o éticas relativas a, por ejemplo, la organización del trabajo. Con todo, la nueva territorialidad de lo peruano en Santiago configura un referente identitario para la población migrante, un locus de recursos para su reproducción económica, mientras simultáneamente introduce un vector de heterogeneidad en el conjunto urbano.

Agradecimientos: A Menara Guizardi, por la paciente lectura y comentario de los borradores de este manuscrito. A los evaluadores de este manuscrito, por sus críticas y sugerencias.

\section{Referencias Citadas}

Anderson, K. 1987. The idea of Chinatown: the power of place and institutional practice in the making of a racial category. Annals of the Association of American Geographers 77(4):580-598.

Appadurai, A. 2001. La Modernidad Desbordada. Dimensiones Culturales de la Globalización. Trilce-FCE, Montevideo.

Araujo, K., M. Legua y L. Ossandón 2000. Migrantes Andinas en Chile. El caso de la Migración Peruana. Fundación Instituto de la Mujer, Santiago.

Beltrán, J., L. Oso y N. Ribas 2007. Empresariado Étnico en España. Fundación CIDOB, Barcelona.

Berg, U. y K. Paerregaard 2005. El Quinto Suyo. Transnacionalidad y Formaciones Diaspóricas en la Migración Peruana. Instituto de Estudios Peruanos, Lima.

Bonacich, E. 1973. A theory of middleman minorities. American Sociological Review 38:583-594.

Boruchoff, J. 1999. Equipaje cultural: objetos, identidad y transnacionalismo en Guerrero y Chicago. En Fronteras Fragmentadas, editado por G. Mummert, pp. 499-517. Colmich, México.

Buckley, M. 1998. Inmigración y comercio en Madrid. Nuevos negocios para nuevas gentes. Anales de Geografía de la Universidad Complutense 18:283-297.

Cano, V. y M. Soffia 2009. Los estudios sobre migración internacional en Chile: apuntes y comentarios para una agenda de investigación actualizada. Papeles de Población 61: 129-167.

Cano, V., M. Soffia y J. Martínez 2009. Conocer para legislar y hacer política: los desafíos de Chile ante un nuevo escenario migratorio. CEPAL Serie Población y Desarrollo 88:1-82.

Cebrián de Miguel, J. y M.I. Bodega 2002. El negocio étnico, nueva fórmula de comercio en el casco antiguo de Madrid. El Caso de Lavapiés. Estudios Geográficos LXIII 248/249:559-580.

de Certeau, M. 2000. La Invención de lo Cotidiano 1: Artes de Hacer. Universidad Iberoamericana, México.
Delgado, M. 1999. El animal Público. Hacia una Antropología de los Espacios Urbanos. Anagrama, Barcelona.

- - - 2007. Sociedades Movedizas. Pasos hacia una Antropología de las Calles. Anagrama, Barcelona.

Ducci, M.E. y L. Rojas 2010. La pequeña Lima: nueva cara y vitalidad para el centro de Santiago de Chile. Eure 36(108):95-121.

Foucault, M. 2002. Vigilar y Castigar: Nacimiento de la Prisión. Ediciones Siglo XXI, Buenos Aires.

Garcés, A. 2005. Espacios comerciales. En Espacios Urbanos e Inmigración en el Madrid del Siglo XXI. VV.AA., pp. 81-148. Casa Encendida, Madrid.

- - - 2006. Configuraciones espaciales de lo inmigrante: usos y apropiaciones de la ciudad. Papeles del CEIC $\mathrm{N}^{\circ} 20$ : 1-34. http://www.identidadcolectiva.es/pdf/20.pdf

- - - 2007. Entre lugares y espacios desbordados: formaciones urbanas de la migración peruana en Santiago de Chile. Serie Documentos. Facultad de Ciencias Sociales - Universidad Central No 2:5-22. http://www.fcsucentral.cl/varios/files/file/ publicaciones/antropologiaok.pdf

- - - 2010. Movimientos y localizaciones: espacios públicos y economías de la migración peruana en Santiago de Chile. Tesis Doctoral. Departamento de Antropología Social, Universidad Autónoma de Madrid, Madrid.

Glick Schiller, N., L. Basch y C. Szanton Blanc 1992. Transnationalism: A new analytic framework for understanding migration. En Towards a Transnational Perspective of Migration. Race, Class, Ethnicty and Nationalism Reconsidered, editado por N. Glick Schiller, L. Basch y C. Blanc-Szanton, pp. ix-xiv. Annals New York Academy of Sciences, New York.

Golte, J. 1999. Redes étnicas y globalización. Revista de Sociología, Volumen 11, Número 12. http://www.cholonautas. edu.pe/modulo/upload/golte3.pdf (1 de diciembre de 2010) 
Kloosterman, R., J. Van der Leun y J. Rath 1999. Mixed- embeddedness: (In)formal economic activities and immigrant businesses in the Netherlands. International Journal of Urban Research 23 (2):253-267.

Light, I. y S. Gold 2000. Ethnic Economies. Academic Press, San Diego.

Luque, J. 2007. Asociaciones políticas de inmigrantes peruanos y la "Lima Chica" en Santiago de Chile. Migraciones Internacionales 4(2):121-150.

Martínez Pizarro, J. 2003. El encanto de los datos. Sociodemografía de la inmigración en Chile según el censo de 2002. CEPAL. Serie Población y Desarrollo 49:1-60.

Martínez Veiga, U. 1999. Pobreza, Segregación y Exclusión Espacial. La Vivienda de los Inmigrantes Extranjeros en España. Icaria, Barcelona.

- - - 2008. Inseguridad habitacional, segregación espacial e higiene. En La Inmigración en la Sociedad Española: una Radiografía Multidisciplinar, editado por J. García Roca y J. Lacomba, pp. 463-478. Bellaterra, Barcelona.

Massey, D. 1994. A global sense of place. En Space, Place and Gender, editado por D. Massey, pp. 146-156. Polity Press, Cambridge.

Massey, D.S., J. Arango, G. Hugo, A. Kouaouci, A. Pellegrino y J.E. Taylor 1998. Una evaluación de la teoría de la migración internacional: el caso de América del Norte. En Cruzando Fronteras. Migraciones en el Sistema Mundial, compilado por G. Malgesini, pp. 189-264. Icaria, Madrid.

Murunga, G. 2005. "Inherently Unhygienic Races": Plague and the Origins of Settler Dominance in Nairobi, 1899-1907. En African Urban Spaces in Historical Perspective, editado por S.J. Salm y T. Falola, pp. 98-130. University of Rochester, Rochester.

Nightingale, C. 2006. The transnational contexts of early twentieth-century American urban segregation. Journal of Social History 39:667-702.

Peñaranda, M.C., A. Vitores, L. Martínez, J. Muñoz y L. ÍniguezRueda 2011. El acceso público a las tecnologías de la información y la comunicación: el lugar de los locutorios en los procesos migratorios. Papeles del CEIC, vol. 2011/1, No 70, CEIC (Centro de Estudios sobre la Identidad Colectiva), Universidad del País Vasco, http://www.identidadcolectiva.es/pdf/70.pdf.

Portes, A. y L. Jensen 1989. The enclave and the entrants: patterns of ethnic enterprise in Miami before and after Mariel. American Sociological Review 54:929-949.

Portes, A., L. Guarnizo y P. Landolt 1999. The study of transnationalism: pitfalls and promise of an emergent research field. Ethnic and Racial Studies 22 (2):217-237.

Serra, P. 2008. Territorios étnicos urbanos y negocios étnicos. Simposio Internacional "Nuevos Retos del Transnacionalismo en el Estudio de las Migraciones, Universidad Autónoma de Barcelona. http://docsgedime.files.wordpress.com/2008/02/ tc-pau-serra.pdf

Signorelli, A. 1999. Antropología Urbana. Anthropos, México. Solé, C. y S. Parella 2005. Negocios Étnicos. Los Comercios de los Inmigrantes No Comunitarios en Cataluña. CIDOB, Barcelona.

Stefoni, C. 2002. Inmigración Peruana en Chile: Una Oportunidad a la Integración. Editorial Universitaria, Santiago.

- - - 2005. Comunidades transnacionales y la emergencia de nuevas oportunidades económicas. De empleados a microempresarios. Persona y Sociedad XIX (3):183-197.

Tarrius, A. 2000. Leer, describir, interpretar. Las circulaciones migratorias: conveniencia de la noción de "territorio circulatorio". Los nuevos hábitos de la identidad. Relaciones 83 XXI:38-66.

Waldinger, R., H. Aldrich y R. Ward 1990. Ethnic Entrepreneurs. Immigrant Business in Industrial Societies. SAGE Publications, Newbury Park.

Werbner, P. 1987. Enclave economies and family firms: Pakistani traders in a British city. En Migrants, Workers, and the Social Order, editado por J. Eades, pp. 213-233. Tavistock, Nueva York y Londres.

Wilson, K. y A. Portes 1980. Immigrant enclaves: An analysis of the labour markets experiencies of Cubans in Miami. American Journal of Sociology 86:295-319.

\section{Notas}

1 Nos referimos a la calle Rivera y alrededores en la comuna de Independencia, y a la calle Catedral y alrededores en la comuna de Santiago, espacios que fueron seleccionados por presentar la referida concentración residencial y económica. El trabajo de campo fue realizado en distintas fases entre los años 2005 y 2009, e incluyó principalmente el desarrollo de observación participante y entrevistas con migrantes peruanos usuarios de los espacios y con comerciantes tanto de origen peruano como chileno que desarrollan su actividad económica en los mismos espacios.

2 Stefoni detectaba, ya en 2005, indicios de este desplazamiento laboral desde el empleo asalariado hacia la conformación de pequeñas empresas por parte de los migrantes peruanos (Stefoni 2005).
3 Entendidos éstos como aventuras narrativas organizadoras del espacio, como producción de unas geografías de acciones, que "hacen el viaje antes o al mismo tiempo que los pies lo ejecutan" (de Certeau 2000).

4 Es interesante mencionar aquí la festividad religiosa del Señor de los Milagros, evento que congrega en el centro de Santiago a importante parte de la comunidad peruana, y que ha sido estudiado en distintos contextos como un rasgo que caracteriza a las migraciones peruanas (Berg y Paerregaard 2005; Ducci y Rojas 2010).

5 Algunos trabajos acerca de las organizaciones políticas de peruanos en Santiago han intentado describir la emergencia de un transnacionalismo político vinculado a la formación de enclaves territoriales en Santiago (Luque 
2007). Sin embargo es necesario relativizar la magnitud de este tipo de lazos, sobre todo si se piensa en el perfil fundamentalmente laboral y/o económico del flujo en cuestión.

6 Los delitos de mayor connotación social refieren a los de hurto, lesiones, robo con fuerza, robo con intimidación, robo con violencia, robo por sorpresa, violación y homicidio.

7 Nos referimos al intervalo de tiempo que va desde el primer semestre de 2006 al primer trimestre de 2007, período en el que las denuncias pasan de 174 a 282 en el caso del espacio de calle Catedral, y de 96 a 139 en el espacio de calle Rivera (datos de la División de Seguridad Pública-Ministerio del Interior, Gobierno de Chile).

8 Es importante tener en cuenta que las localizaciones observables pueden responder también a otros fenómenos que actúan simultáneamente. Por ejemplo, en el caso del centro de Santiago coincide con la decadencia de una zona comercial y su revitalización a partir de las distintas actividades económicas a que da origen la migración. 
